\title{
MiR-369-3p participates in endometrioid adenocarcinoma via the regulation of autophagy
}

Ping Liu ${ }^{1,2}$, Chengbin Ma², Qiongwei Wu², Wenying Zhang ${ }^{2}$, Cao Wang ${ }^{2}$, Li Yuan ${ }^{3 *}$ and Xiaowei Xi ${ }^{1 *}$

\begin{abstract}
Background: The aim of this study is to examine miRNA profiling and miR-369-3p participates in endometrioid adenocarcinoma (EEC) via the regulation of autophagy.

Methods: EEC and its adjacent normal tissues were obtained from 20 clinical patients after surgery. MiRNA profiling was performed using next generation sequencing (NGS) and was validated with quantitative real time PCR (qRT-PCR). qRT-PCR was also employed to measure miR-369-3p and autophagy-related protein 10 (ATG10) expression levels. Western blotting assay was performed to measure the expressions of ATG10 and LC3B. Luciferase reporter assay was conducted to confirm the direct targeting of ATG10 by miR-369-3p. Cell proliferation and migration assays were utilized to analyze the role of miR-369-3p in HEC-1-A cells.
\end{abstract}

Results: We found that miR-369-3p expression levels were down-regulated in EEC compared to the control tissues. The overexpression of miR-369-3p inhibited cell proliferation and migration in EEC; furthermore, ATG10 expression increased in EEC tissues. ATG10 was found to be a potential target of miR-369-3p via a dual-luciferase reporter assay, and ATG10 was shown to be down-regulated by miR-369-3p in protein levels.

Conclusions: This study revealed that miR-369-3p inhibited cell proliferation and migration by targeting ATG10 via autophagy in EEC.

Keywords: Endometrioid adenocarcinoma, MicroRNA, Autophagy, ATG10

\section{Background}

Endometrial adenocarcinoma (EEC) is a common and highly malignant gynecological tumor [1]. In recent years, its incidence rate in China and abroad has shown an upward trend, with the prognosis of EEC being poor and the improvement of prognosis depending on early diagnosis [2]. However, the early diagnosis of EEC is challenging. Specifically, identifying highly sensitive and specific tumor markers is a challenge that needs to be solved for the early diagnosis of this cancer.

\footnotetext{
*Correspondence: jiuyuehanguang@163.com; xixiaowei1966@126.com ${ }^{1}$ Gynecology Department, Shanghai General Hospital of Nanjing Medical University, Shanghai 200080, China

${ }^{3}$ School of Basic Medical Sciences of Nanjing Medical University, Nanjing 211166, China

Full list of author information is available at the end of the article
}

Autophagy is an important catabolic process in which damaged organelles or protein recycling undergoes nutrient starvation or stress [3], and it is required for glucose homeostasis and lung tumor maintenance [4]. Autophagy participates in a critical pathway that maintains intracellular balance in regard to both the initiation and prevention of cancer [5]. It also supports the survival of tumor cells under metabolic and therapeutic stress [6]. Furthermore, the inhibition of autophagy can increase the chemo sensitivity of cancer cells $[7,8]$. However, autophagy is a double-edged sword when regarding health and disease [9]. Autophagy begins with autophagosome formation and concludes with a completed autophagolysosome [10]. In addition, the synthesis of the autophagosome complex is regulated by autophagy-related genes (ATG) [11-13]. Several important genes are involved in the autophagy process, including ATG3, ATG5, ATG7, ATG10, ATG12,

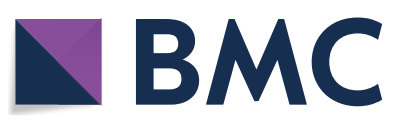

(c) The Author(s) 2019. This article is distributed under the terms of the Creative Commons Attribution 4.0 International License (http://creativecommons.org/licenses/by/4.0/), which permits unrestricted use, distribution, and reproduction in any medium, provided you give appropriate credit to the original author(s) and the source, provide a link to the Creative Commons license, and indicate if changes were made. The Creative Commons Public Domain Dedication waiver (http://creativecommons.org/ publicdomain/zero/1.0/) applies to the data made available in this article, unless otherwise stated. 
and LC310. More evidence has also highlighted the importance of these aforementioned autophagy-related genes in cancer maintenance, clinical therapy, and pathogenesis 8. ATG10 is an E2-like enzyme involved in two ubiquitin-like modifications essential to autophagosome formation [14]. Increased expression of ATG10 in colorectal cancer is associated with lymphovascular invasion and lymph node metastasis [15]. Through the genetically engineered mouse models, ATG7 ablation was found to block tumor growth and induce tumor cell death in lung cancer, indicating that autophagy inhibition might serve as a promising strategy for lung cancer therapy [12]. However, the mechanisms and clinical value of these critical regulatory autophagy-related genes have not been explored in regard to EEC.

MicroRNAs (miRNAs or miRs) are classified as small, endogenous, non-coding, single-stranded RNAs that regulate target genes post-transcriptionally by specifically binding to the $3^{\prime}$ untranslated region ( $3^{\prime}-\mathrm{UTR}$ ) of the target mRNA [16]. It was reported that miRNAs play an important role in various biological processes, including cell growth, cell death, apoptosis, and tumorigenesis [17, 18]. Several studies using miRNA microarray analyses or miRNA-Seq have revealed that the expression of miR369-3p increases in the skin tissues of psoriasis patients [19] and that the aberrant expression of miR-369-3p correlates with disease severity in hepatocellular carcinoma [20]. Hu et al. [21] reported that miR-369 induced cellular reprogramming and regulated malignant phenotypes of human colorectal cancer. $\mathrm{Li}$ et al. found that miR369-3p was differently expressed in Hirschsprung disease and may play a crucial role in its pathology [22]. Agarwal et al. [23] indicated that the upregulation of miR-369-3p suppresses cell migration and proliferation by targeting SOX4 in Hirschsprung's disease. However, the detailed mechanism of miR-369-3p and its potential target gene involved in the pathogenesis of endometrial adenocarcinoma is still not widely understood.

Therefore, we sought to determine whether miR$369-3 p$ is involved in the pathology of EEC and whether miR-369-3p contributes to EEC by regulating autophagy.

\section{Methods}

\section{Experimental subjects}

This is a retrospective study of EEC tissues and their adjacent non-cancerous counterparts regarding four patients treated by surgical resection. The EEC patients were admitted to the Shanghai First People's Hospital from July 2017 to July 2018. None of the patients underwent chemotherapy, radiotherapy, or steroid treatment. This study was approved by the Review Board of the Shanghai First People's Hospital under the supervision of the Ethics Committee, and all patients signed the informed consent forms. This study's protocol conformed to the ethical guidelines of the 1975 Declaration of Helsinki. In this study, EEC and their adjacent normal tissues were obtained from 20 clinical patients. Four pairs were used for next generation sequencing (NGS) detection, and the others were used for qRT-PCR or Western blot (WB) detection. Diagnosis of EEC was based upon clinical, imaging, and histopathological findings based on explant evaluation according to the revised WHO classification of tumors. Patients were aged $40-45$ years, with a mean age of 43.8 years. The EEC stage, in accordance with the surgery-pathological staging criteria issued in 1988 by the International Federation of Gynecology and Obstetrics (FIGO), was identified as III-IV. Pathological records and tissue samples were collected during surgery and immediately snap-frozen in liquid nitrogen. The clinical characteristics of the patients are presented in Table 1.

\section{Cell culture}

HEC-1-A cells (human EEC cells-1-A) are human EEC cell lines and were obtained from the American Type Culture Collection (Manassas, VA, USA) and cultured in McCoy's 5A modified medium supplemented with $10 \%$ fetal bovine serum and 1\% penicillin/streptomycin (Sigma-Aldrich, St Louis, MO, USA). HEC-1-A cells are used as an accepted model for studying endometrial cancer [22]. MiR-369-3p, a mimic, inhibitor, agomir or antagomir, and an agomir negative-treated control (NTC) (final concentration, $2.5 \mathrm{nmol} / \mathrm{L}$ ) were purchased from RiboBio Inc. (GuangZhou, China). An adenovirus (Ad)-expressing system, used for the overexpression the ATG10 protein (Ad-expression ATG10), was purchased from Hanbio, Shanghai, China, with an Ad-expressing green fluorescent protein (GFP) serving as a control. Cells were treated with miR-369-3p agomir, antagomir, or NTC in six-well plates following manufacture protocol. For the rescue experiments, cells were treated with both miR-369-3p agomir and Ad-expression ATG10.

\section{MicroRNA sequencing}

MiRNAs were isolated using the miRNA kit (Qiagen, Hilden, Germany) according to the manufacturer instructions. The quantity and quality of RNA were assessed using the NanoDrop ND-1000 instrument (Nanodrop

Table 1 Clinical characteristics of the patients with EEC

\begin{tabular}{ll}
\hline Sex (female) & 20 \\
Mean age & $43.8 \pm 1.3$ \\
Stage (FIGO) & $1 \mathrm{a}+1 \mathrm{~b}$ \\
TNM Classification & $\mathrm{I} / \mathrm{Il}$ \\
Nodules metastases & No \\
\hline
\end{tabular}


Technologies, Wilmington, USA) and the Bioanalyser 2100 system (Agilent Technologies, CA, USA) using the Agilent RNA 6000 Nano according to the manufacturer instructions. MiRNAs were profiled by NGS on the Illumina Hiseq 2000 instrument (Illumina Inc., San Diego, USA) using the Illumina TruSeq Rapid SBS preparation protocol (Arraystar Inc., Rockville, USA). The cDNA library was prepared using the SMART cDNA Library Construction Kit (Clontech Laboratories, Inc. CA, USA) according to the manufacturer instructions. The quantification of the cDNA library was measured using a 2100 Bioanalyser (Agilent Technologies), with the final concentration being $10 \mathrm{pM}$. Differentially expressed miRNAs between the two groups were analyzed using the twotailed, homoscedastic $t$-test.

\section{Validation test of quantitative real time PCR (qRT-PCR)}

qRT-PCR was performed to validate the results of the microRNA sequencing. RNA extraction was conducted as previously described. Reverse transcription was conducted using a QuantiTect Reverse Transcription Kit (Qiagen, Germany) according to the manufacturer instructions. The PCR reaction system was created using a total volume of $20 \mu \mathrm{L}$ containing $10 \mu \mathrm{L}$ of $2 \times$ master mix, $8 \mu \mathrm{L}$ of diluted cDNA, and $0.5 \mu \mathrm{L}$ of each of the forward and reverse primers, respectively. The PCR was completed in following steps: step $1,95^{\circ} \mathrm{C}$ for $3 \mathrm{~min}$; step 2, $95{ }^{\circ} \mathrm{C}$ for $15 \mathrm{~s}$ and $60{ }^{\circ} \mathrm{C}$ for $1 \mathrm{~min}$ (repeat step 2 for 40 cycles). The GAPDH gene was used as the reference gene, and the $2^{-\Delta \Delta C t}$ method was used to calculate the relative level of gene expression in EEC and its adjacent non-cancerous counterparts.

\section{MiR-369-3p target prediction and functional enrichment analysis}

mRNA gene targets were identified using TargetScan (v6.2) [24], Miranda, and Microcosm v5 [25]. The Venny tool (Venny v2.0.2) [26] was used to filter miRNA target genes into all three programs. Gene ontology (GO) (TopGO v4.2) grouping categories (Biological Process, Cellular Components, and Molecular Processes) and the Kyoto Encyclopedia of Genes and Genomes (KEGG) pathway analysis (Arraystar Inc., Rockville, USA) were used to perform the functional analysis for predicted miRNA target genes.

\section{Luciferase assay}

The mRNA 3'-UTR, containing the putative target site for miR-369-3p, was amplified from genomic DNA via PCR amplification and inserted into the pMIR-REPORT (RiboBio, Guangzhou, China). A mutant reporter plasmid of the miR-369-3p complementary locus was created with the Quik-Change II Site-Directed Mutagenesis Kit (Stratagene, La Jolla, CA, USA) according to the manufacturer instructions. The HEC-1-A cells were transiently transfected with wild-type or mutant reporter plasmid. Luciferase activity was measured $24 \mathrm{~h}$ after transfection using the luciferase activity kit (BioVision, San Francisco, CA, USA) according to the manufacturer instructions. Three independent experiments were performed in triplicate.

\section{Western blot (WB) assay}

The total protein from cells was extracted with RIPA lysate. The homogenates, which contained $20 \mu \mathrm{g}$ of protein, were run on 12-15\% SDS-PAGE gels and then transferred to a PVDF membrane (Millipore, Shanghai, China). The PVDF membrane was blocked with $5 \%$ nonfat dry milk for $1 \mathrm{~h}$ at room temperature and incubated overnight at $4{ }^{\circ} \mathrm{C}$ with primary antibodies, including LC3 (1:300; Abcam), Atg10 (1:1000; GTX), and GAPDH (1:3000; Abcam).

\section{Cell proliferation}

Cell Counting Kit-8 (CCK-8, Dojindo Laboratories, Kumamoto, Japan) was used to detect cell proliferation.

Table 2 Differentially expressed microRNAs identified by next generation sequencing

\begin{tabular}{|c|c|c|c|c|c|c|}
\hline \multicolumn{3}{|c|}{ miRNA information } & \multicolumn{4}{|c|}{ Statistics and regulation } \\
\hline Mature-miRNA & Pre-miRNA & Mature-sequence & Fold change & $p$-value & FDR & Regulation \\
\hline hsa-miR-136-3p & hsa-mir-136 & CAUCAUCGUCUCAAAUGAGUCU & 63.72 & 0.005402 & 0.035995 & Down \\
\hline hsa-miR-369-3p & hsa-mir-369 & AAUAAUACAUGGUUGAUCUUU & 150.75 & 0.009838 & 0.053134 & Down \\
\hline hsa-miR-451a & hsa-mir-451a & AAACCGUUACCAUUACUGAGUU & 69.29 & 0.048753 & 0.143407 & Down \\
\hline hsa-miR-493-5p & hsa-mir-493 & UUGUACAUGGUAGGCUUUCAUU & 90.00 & 0.019385 & 0.081725 & Down \\
\hline hsa-miR-543 & hsa-mir-543 & AAACAUUCGCGGUGCACUUCUU & 90.40 & 0.035484 & 0.117148 & Down \\
\hline hsa-miR-10b-5p & hsa-mir-10b & UACCCUGUAGAACCGAAUUUGUG & 3.52 & 0.012481 & 0.061076 & Up \\
\hline hsa-miR-143-3p & hsa-mir-143 & UGAGAUGAAGCACUGUAGCUC & 9.21 & $4.05 E-08$ & 0.000012 & Up \\
\hline hsa-miR-145-5p & hsa-mir-145 & GUCCAGUUUUCCCAGGAAUCCCU & 2.89 & 0.012701 & 0.061076 & Up \\
\hline
\end{tabular}


hsa-miR-136-3p

hsa-miR-369-3p
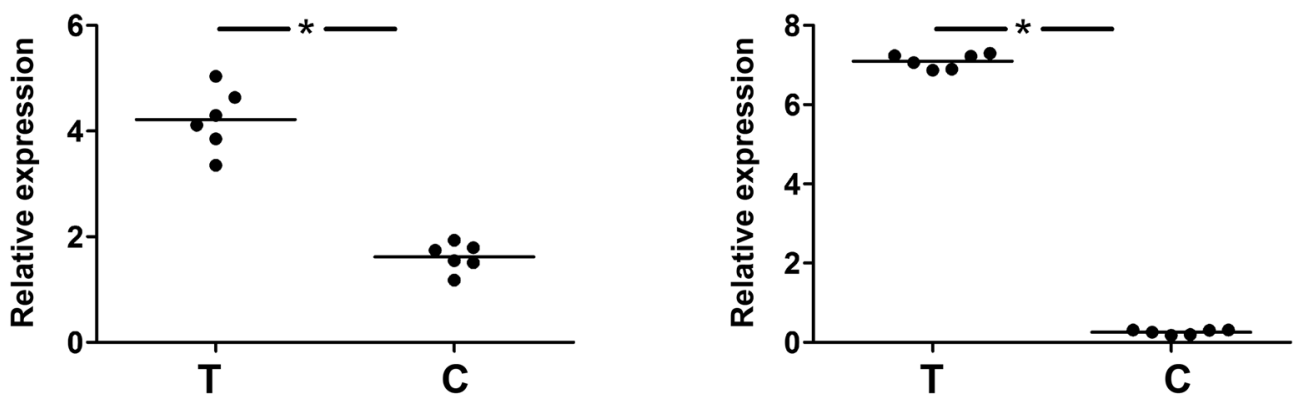

hsa-miR-451a
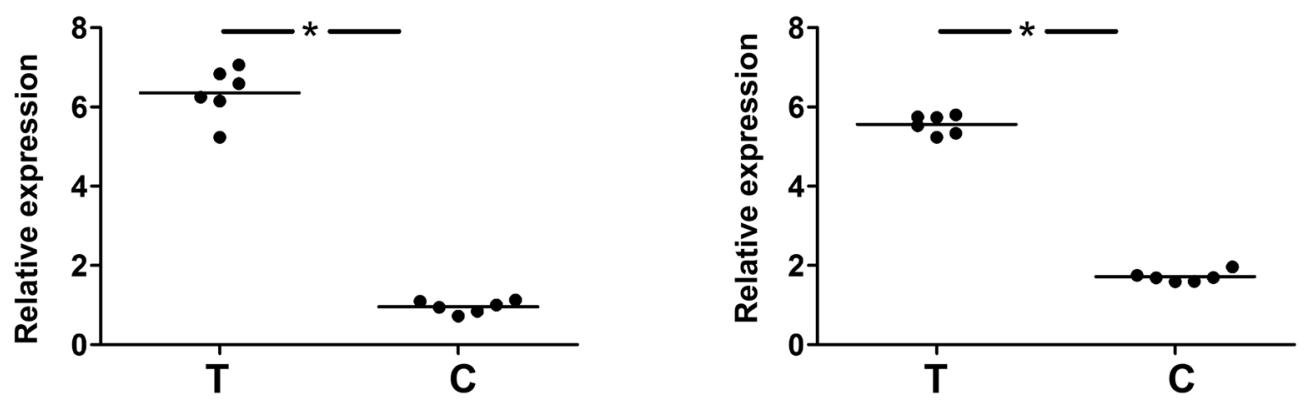

hsa-miR-136-3p

hsa-miR-136-3p
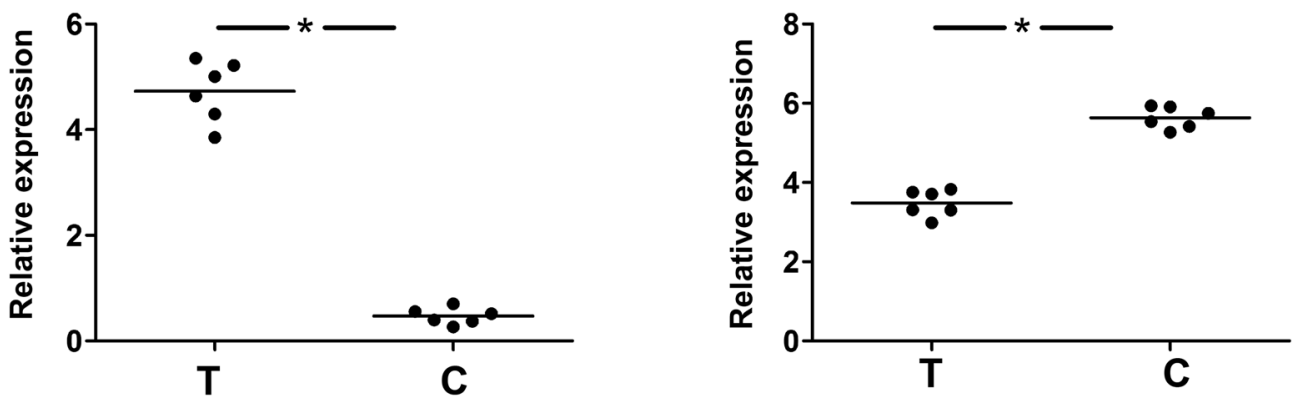

hsa-miR-143-3p

hsa-miR-145-5p
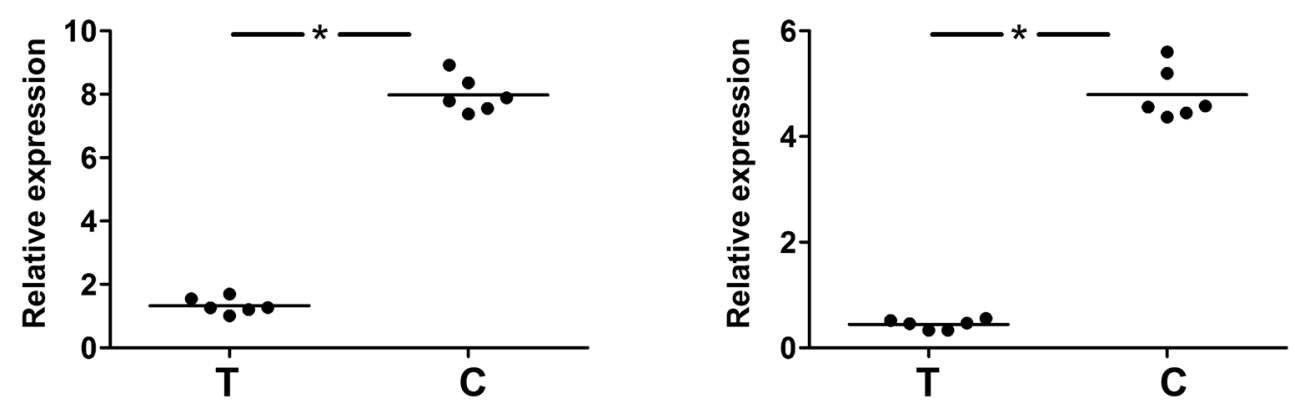

Fig. 1 Results of quantitative real time PCR (qRT-PCR). qRT-PCR was used to validate the results of small RNA-Sequencing. The qRT-PCR results demonstrated an increase in the expression of hsa-miR-10b-5p (6.50 \pm 0.09 -fold), hsa-miR-143-3p (2.70 \pm 0.16 -fold), and hsa-miR-145-5p $(5.60 \pm 0.22$-fold $)$ in the EEC tissues, whereas the expression of hsa-miR-369-3p $(-7.50 \pm 0.24$-fold), hsa-miR-136-3p $(-3.72 \pm 0.14$-fold), hsa-miR-451a ( $-2.69 \pm 0.13$-fold), hsa-miR-493-5p (5.60 \pm 0.21 -fold), and hsa-miR-543 ( $-4.51 \pm 0.16$-fold) decreased in the EEC tissues, respectively. These data were consistent with the small RNA-sequencing results. T, EEC tissues; $C$, their adjacent no cancerous counterparts. ${ }^{*} p<0.05$ 


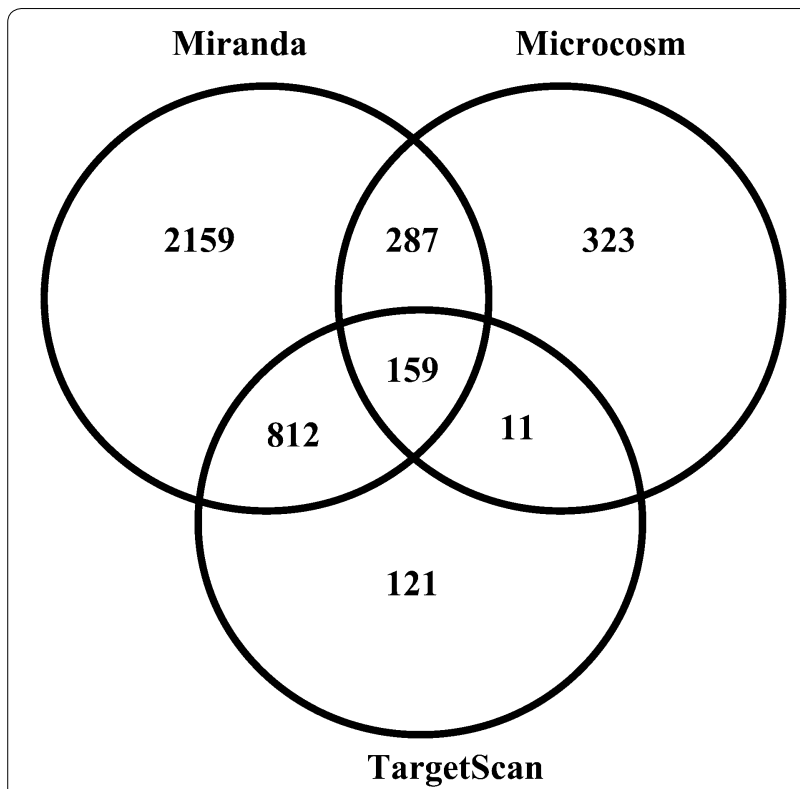

Fig. 2 Venn diagram showing mRNA targets for miR-27b predicted using TargetScan, Miranda, and Microcosm. More than 3000 mRNA targets for miR-27b were predicted by each program. Of these, 742 mRNA targets were commonly predicted by TargetScan and Miranda, 261 mRNA targets were commonly predicted by Miranda and Microcosm, 15 mRNA targets were commonly predicted by Microcosm and TargetScan, and 133 mRNA targets were commonly predicted by TargetScan, Miranda, and Microcosm

HEC-1-A cells treated with miR-369-3p agomir, antagomir, miR-NTC, and Ad-expression ATG10 were briefly seeded into 96 -well plates $\left(5 \times 10^{3}\right.$ cell/well $)$ with $100 \mu \mathrm{l}$ medium. CCK-8 reagents were added into each well at $24 \mathrm{~h}, 48 \mathrm{~h}$, and $72 \mathrm{~h}$ and incubated at $37{ }^{\circ} \mathrm{C}$. The optical density (OD) measured at $450 \mathrm{~nm}$ on an enzyme-linked immunosorbent assay reader. Three independent experiments were performed in triplicate.

\section{Cell migration}

24-well culture plates inserted with 8-mm pore size culture (Transwell; Falcon, BD Biosciences) were used to performed the cell migration assays. The lower chamber was filled with $600 \mu \mathrm{l} \mathrm{McCoy's} \mathrm{5A} \mathrm{modified} \mathrm{medium}$ with $10 \%$ FBS. Cells $\left(1 \times 10^{5}\right.$ cell/well $)$ were seeded to the upper chamber. After $24 \mathrm{~h}$ incubation, the number of the bottom well cells were counted by counting chamber.

\section{Statistical analysis}

All statistical analyses were conducted using GraphPad Prism software 5.0 (GraphPad Software, Inc., San Diego, CA). The quantitative data were expressed as the mean \pm standard error difference $(\overline{\mathrm{X}} \pm \mathrm{SED})$, and a paired $t$-test was used for comparisons between the two groups. A value of $p<0.05$ was considered to be statistically significant. Statistical significance for GO terms was calculated using Fisher's exact test. The significance of KEGG pathways was determined using the EASE-score, Fisher $p$ value, and the Hypergeometric test adjusted for the false discovery rate (FDR).

\section{Results \\ MicroRNA expression profiling involved in EEC samples}

To study the full spectrum of miRNAs involved in EEC, small RNA-sequencing was performed, and 296 of the miRNAs were initially detected. Of these, 8 miRNAs were significantly differentially expressed between EEC tissues and their adjacent non-cancerous counterparts. Five miRNAs (hsa-miR-136-3p, hsa-miR-451a, hsa-493-5p, hsa-369-3p, hsa-miR-543) showed down-regulation, and three miRNAs (hsa-miR-10b-5p, hsa-miR-143-3p, and hsa-miR-145-5p) showed up-regulation between EEC tissues and their adjacent non-cancerous counterparts (Table 2).

\section{Quantitative real time PCR}

qRT-PCR was then used to validate the results of the small RNA-sequencing. The qRT-PCR results demonstrated an increase in the expression of hsa-miR-10b-5p (6.50 \pm 0.09 -fold), hsa-miR-143-3p (2.70 \pm 0.16 -fold), and hsa-miR-145-5p (5.60 \pm 0.22 -fold) in EEC tissues, whereas the expression of hsa-miR-369-3p $(-7.50 \pm 0.24-$ fold), hsa-miR-136-3p ( $-3.72 \pm 0.14$-fold), hsa-miR-451a $(-2.69 \pm 0.13$-fold), hsa-miR-493-5p (5.60 \pm 0.21 -fold), and hsa-miR-543 $(-4.51 \pm 0.16$-fold $)$ decreased in EEC tissues (Fig. 1). These data were consistent with the small RNA-sequencing results.

\section{Target prediction and functional enrichment analysis of miR-369-3p}

Over 2500 putative mRNA targets were predicted for miR-369-3p using TargetScan, Miranda, and Microcosm, of which 159 gene targets were commonly predicted by all three programmes (Fig. 2). Moreover, 812 mRNA targets were commonly predicted by TargetScan and Miranda, 287 mRNA targets were commonly predicted by Miranda and Microcosm, and 11 mRNA targets were commonly predicted by Microcosm and TargetScan.

Functional enrichment analysis identified 427 GO terms enriched by miR-369-3p gene targets, categorized into 17 molecular functions (MF), 253 biological processes (BP), and 17 cellular components (CC) (Additional file 1: Table S1), with the top $10 \mathrm{GO}$ terms of BP illustrated in Fig. 3. Three GO terms, (1) regulation of Ras GTPase activity, (2) regulation of neuroblast proliferation, and (3) regulation of cellular catabolic process, were statistically significant with $\mathrm{p}<0.001, \mathrm{FDR} \leq 0.51$. 


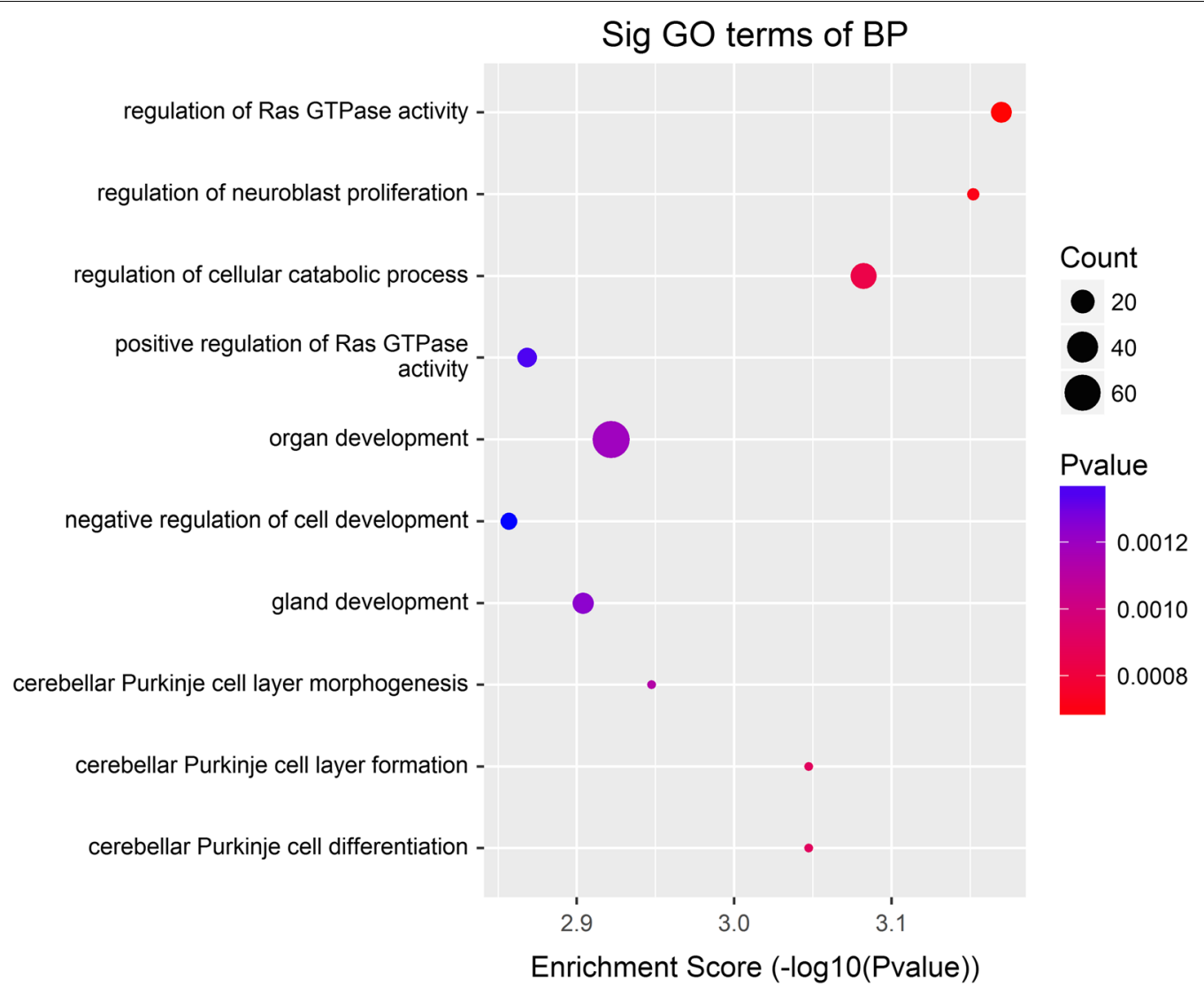

Fig. 3 Top ten GO terms enriched by miR-369-3p gene targets. Data are presented as enriched scores expressed as - $\log 10$ ( $p$ value)

Five significantly enriched signalling pathways (Fisher $\mathrm{p}$ value $\leq 0.05$ ) (Additional file 2: Table S2) were identified. These pathways include the (1) ribosome biogenesis in eukaryotes, (2) regulation of autophagy, (3) measles, (4) tuberculosis, and (5) cytokine-cytokine receptor interaction (Fig. 4).

\section{Autophagy in EEC tissues}

In EEC tissues, the expression of ATG10 was significantly upregulated at the protein levels compared to that of its adjacent non-cancerous counterparts. Furthermore, we found that the expression of LC3B was increased more in EEC tissues than in the adjacent non-cancerous counterparts. This increased LC3B indicated that cancer induced the formation of autophagosomes (Fig. 5).

\section{ATG10 is a direct target of miR-369-3p}

To investigate the molecular mechanism of miR-369-3p in endometrioid adenocarcinoma, we employed a bioinformatics approach using TargetScan, Miranda, and Microcosm software to predict the target genes of miR369-3p. We found that ATG10 may be one of the potential target genes of miR-369-3p. Then, the HEC-1-A cells were treated with miR-369-3p agomir to test the direct link between miR-369-3p and ATG10. We performed a dual-luciferase reporter assay in HEC-1-A cells. After the cells were treated with miR-369-3p, agomir, and the plasmid (pmiR-RBREPORT-ATG10-3'-UTR), a significant decrease in relative luciferase activity was observed $(n=4$ per group; Fig. 6). Furthermore, when the complementary sites in the $3^{\prime}$-UTR of ATG10 were mutant, the suppressive effect of miR-369-3p was abolished due to the interaction between miR-369-3p and ATG10 being disrupted. These results suggest the ATG10 is a direct target of miR-369-3p.

\section{The overexpression of miR-369-3p inhibits EEC cell proliferation and migration via autophagy}

Next, we studied whether the overexpression of miR369-3p can inhibit HEC-1-A cell proliferation and migration by targeting ATG10 in EEC via autophagy. We examined the mRNA and protein expression levels of ATG10 via Western Blot and qRT-PCR after HEC-1-A cell were treated with miR-369-3p agomir or antagomir. Compared with the miR-369-3p agomir group, the mRNA and protein levels of ATG10 were rescued in the miR-369-3p and ATG10 (Fig. 7a, b). Compared to the 


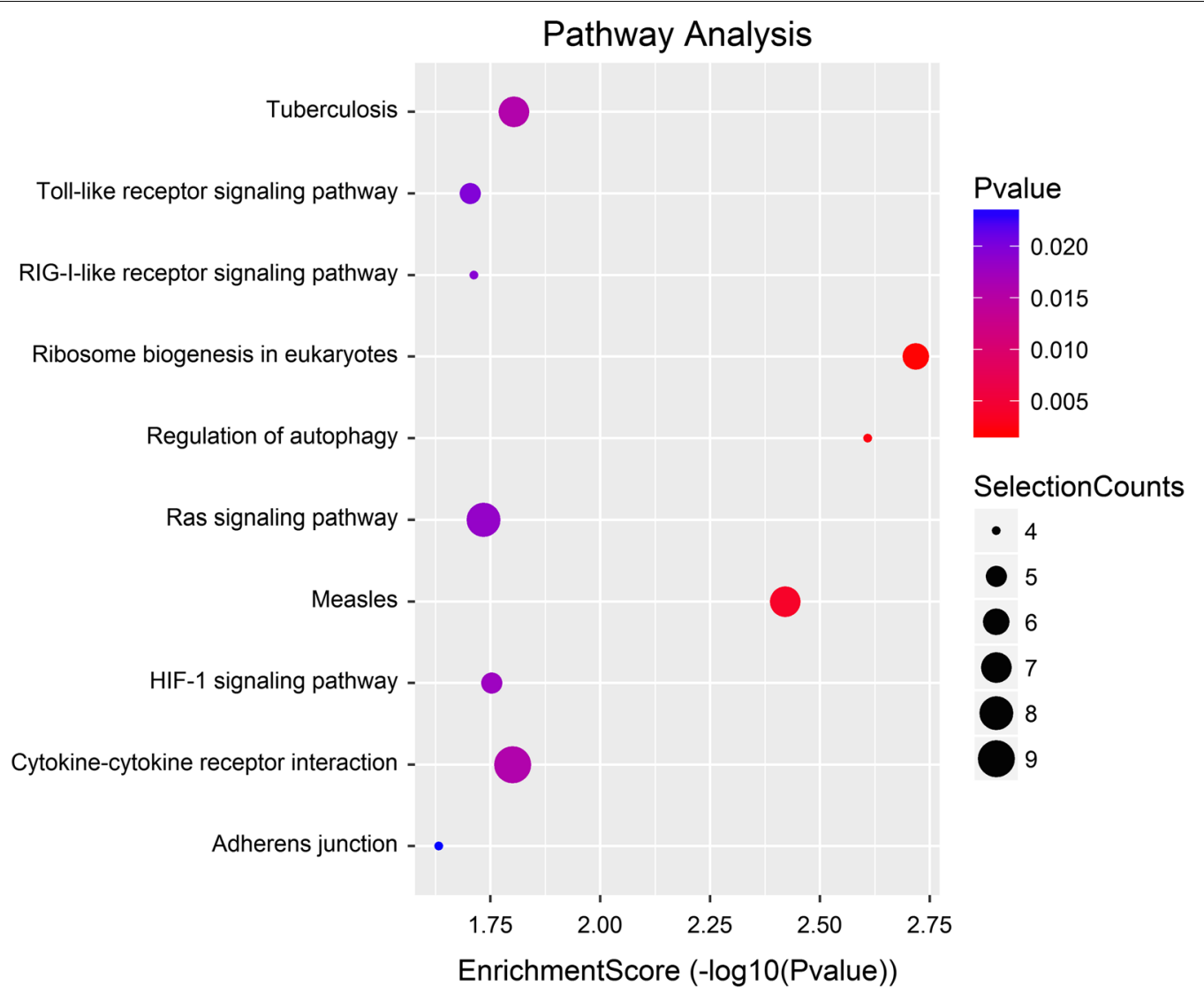

Fig. 4 KEGG pathway analysis of miR-369-3p gene targets. MiR-369-3p gene targets were mapped to KEGG pathways. Data are presented as enriched scores expressed as $-\log 10$ ( $p$ value)

miR-369-3p agomir group, ATG10 overexpression inhibited cell proliferation (Fig. 7c). Then, we investigated the effects of ATG10 on cell migration in HEC-1-A cells via Transwell assay. The overexpression of ATG10 inhibited cell migration when the cells were treated with miR369-3p agomir (Fig. 7d). Thus, miR-369-3p promoted cell proliferation and migration by ATG10 through autophagy in HEC-1-A cells.

\section{Discussion}

In this study, we identified miRNA profiling across EEC tissues and their adjacent non-cancerous counterparts. miRNAs from different tissues were identified and compared using NGS and qRT-PCR. Furthermore, we studied the role of miR-369-3p in EEC tissues because (1) miR-369-3p was the most decreased miRNA in the present study and (2) miR-369-3p has been linked to cancerous elements, such as colorectal cancer cells [27] and papillary thyroid cancer [28]. However, there is still little understanding of miR-369-3p in EEC. Using mRNA target prediction analysis, we found and validated that ATG10 was one of the target genes of miR-369-3p.
Moreover, we found that ATG10 is a critical gene in autophagy and cancer [29].

New biomarkers for the early diagnosis of the increasing prevalence of EEC requires further development. It was reported that miRNAs play important roles in the occurrence and development of EEC. We found that miR-23a regulates the epithelial-to-mesenchymal transition in EEC by targeting SMAD3 [30]. Lee et al. [31] identified the microRNA expression profile related to lymph node status in women with early-stage grade 1-2 endometrial cancer. Wilczynski et al. [32] reported aberrant microRNA expression in endometrial carcinoma using formalin-fixed paraffin-embedded (FFPE) tissues. In this study, we found that miR-369-3p was significantly downexpressed in EEC tissues compared to control tissues and that the upregulation of miR-369-3p inhibited EEC cell proliferation via ATG10.

Several studies have reported that some miRNAs, such as miR-200c [33] and miR-23a [30], can be used as potential therapeutic approaches to EEC. Furthermore, expression levels of miRNA-200c significantly increased in endometrioid endometrial cancer samples. The expression of miRNA-200c was maintained 

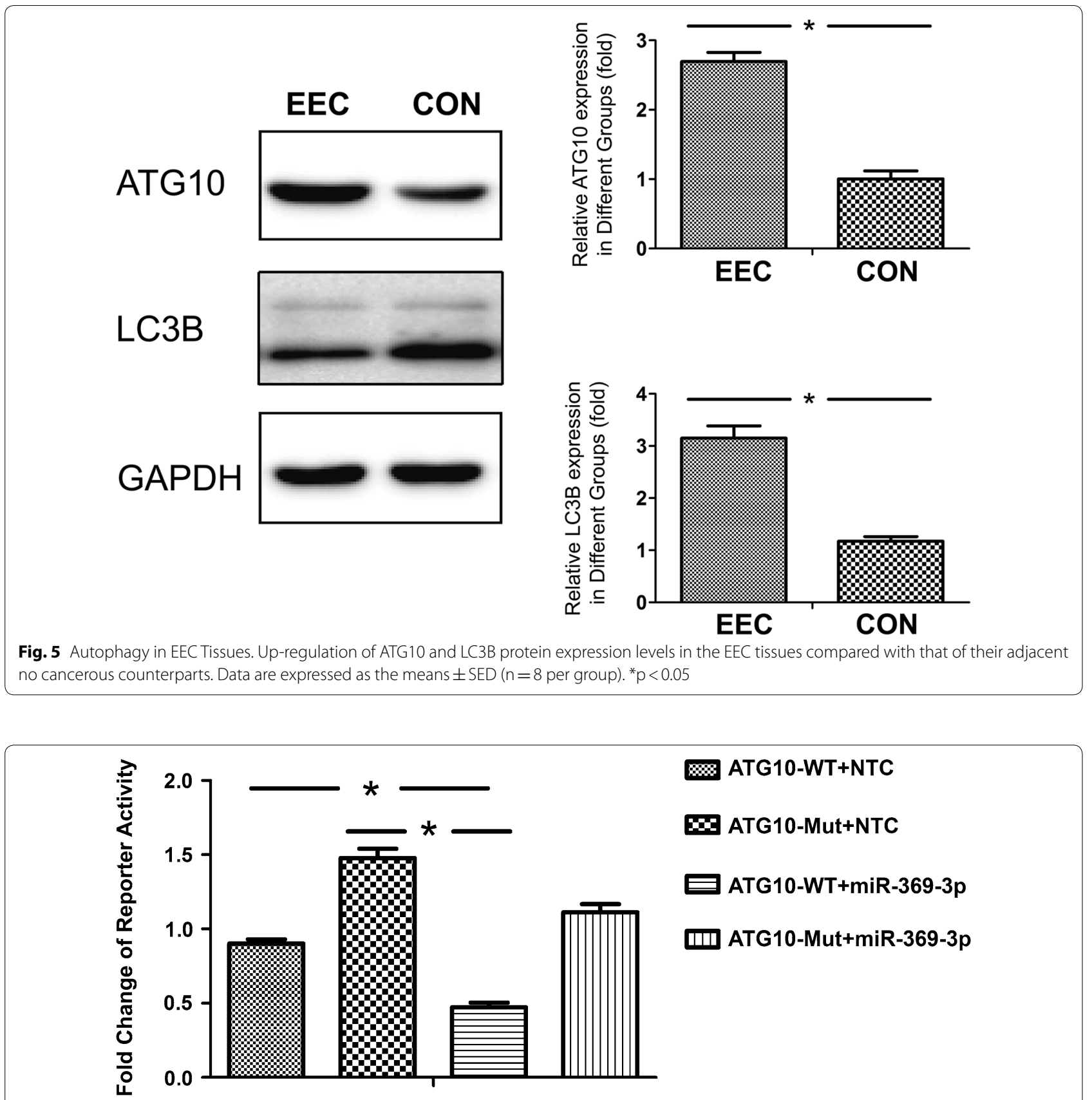

Fig. 6 ATG10 Is a Direct Target of miR-369-3p. miR-369-3p targeting to the ATG10 3'UTR was determined by a luciferase reporter assay in HEC-1-A cells. Luciferase activity was measured $24 \mathrm{~h}$ after transfection. Data are expressed as the mean \pm SED ( $n=4$ per group). ${ }^{*} p<0.05$

at significantly higher levels in early-stage endometrioid endometrial cancer compared to more advanced stages. In our previous study, we found that miR-23a expression was down-regulated in human EEC samples, and the overexpression of miR-23a in HEC-1-A cells increased E-cadherin expression while decreasing the expression of vimentin and alpha smooth muscle actin, markers of the mesenchymal cellular phenotype. In this study, miR-369-3p inhibited the proliferation of HEC-1-A cells. Furthermore, miR-369-3p agomir treatment significantly inhibited the growth of HEC1-A cells. The attenuation of deregulated miR-369-3p expression sensitizes non-small cell lung cancer cells to cisplatin via the modulation of the nucleotide sugar transporter SLC35F5 [33]. It was reported that serum and skin levels of miR-369-3p in psoriasis patients correlated with disease severity [34]. miR-369-3p can inhibit cell proliferation in papillary thyroid cancer by 


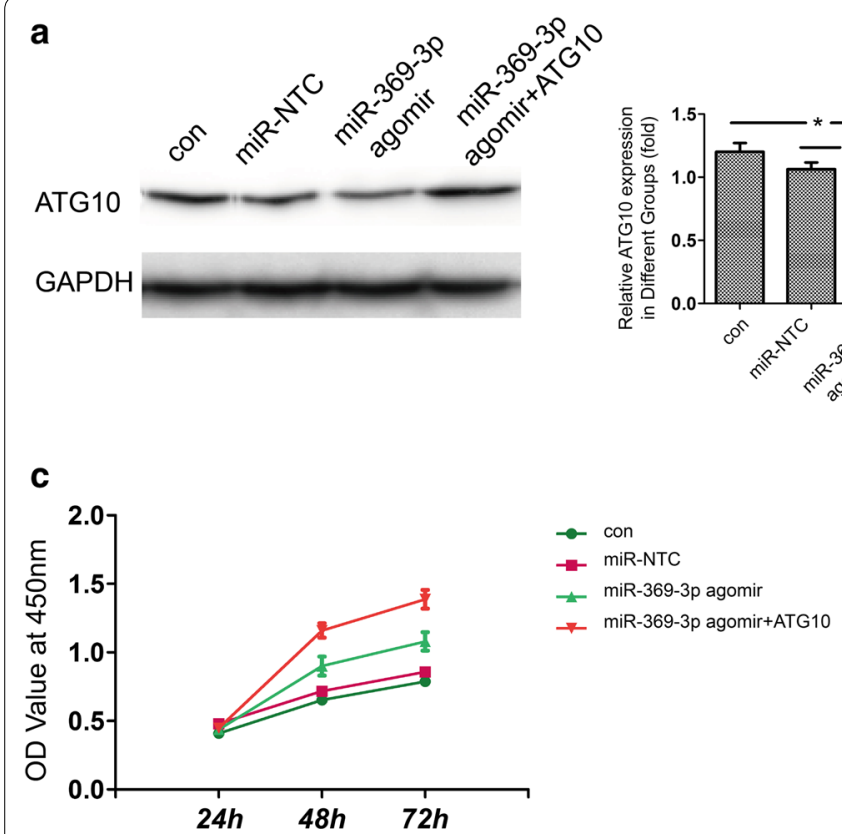

b

Fig. 7 Overexpression of miR-369-3p significantly inhibited HEC-1-A cell proliferation and migration. a The treatment with miR-NTC, miR-369-3p agomir and miR-369-3p agomir + ATG10 cells were validated protein expression levels of ATG10 by Western blot. * $p<0.05$. $\mathbf{b}$ The treatment with miR-NTC, miR-369-3p agomir and miR-369-3p agomir + ATG10 cells were validated mRNA expression levels of ATG10 by qRT-PCR. * $p<0.05 . \mathbf{c} C C K-8$ assay results showed that overexpression of ATG10 suppressed HEC-1-A cell proliferation. ${ }^{*} p<0.05$. $\mathbf{d}$ Cell migration were detected using transwell assay. Migrated cells were counted by counting chamber in each group. ${ }^{*} p<0.05$

down-regulating the target gene, TSPAN13 [27]. Additionally, LncRNA OIP5-AS1 was reported to regulate radio resistance by targeting DYRK1A via miR-369-3p in colorectal cancer cells [26]. The upregulation of miR-369-3p was also found to suppress cell migration and proliferation by targeting SOX4 in Hirschsprung's disease [35]. The aforementioned reports suggest that the up-regulation of miR-369-3p can activate multiple signaling pathways required for cancer or other diseases.

\section{Conclusions}

In summary, our data demonstrated that miR-369-3p was downregulated in endometrioid endometrial cancer. Overexpression of miR-369-3p inhibits cancer cell proliferation by targeting ATG10. This mechanism was related to the autophagy signaling pathways.

\section{Additional files}

Additional file 1: Table S1. Functional enrichment analysis identified $427 \mathrm{GO}$ terms enriched by miR-369-3p gene targets, categorized into 17 molecular functions (MF), 253 biological processes (BP), and 17 cellular components (CC).
Additional file 2: Table S2. Three GO terms, (1) regulation of Ras GTPase activity, (2) regulation of neuroblast proliferation, and (3) regulation of cellular catabolic process, were statistically significant with $p<0.001$, $F D R \leq 0.51$. Five significantly enriched signalling pathways (Fisher $p$ value $\leq 0.05$ ) were identified.

\section{Abbreviations}

EEC: endometrioid adenocarcinoma; CCK-8: cell counting kit-8; miR: microRNA.

\section{Acknowledgements}

The authors want to thank Bing Li (Changning Maternity and Infant Health Hospital) for collecting endometrial carcinoma samples.

\section{Authors' contributions}

$\mathrm{PL}, \mathrm{CM}$ and $\mathrm{XX}$ participated in the conception and design of the study. PL, CM and QW contributed the cell culture and treatment for the study. CM and CW performed the data collection and analysis. PL, CM, QW and LY contributed the critical revision of the manuscript for important intellectual content. PL and XX interpreted the data and produced the draft of the manuscript. All authors read and approved the final manuscript.

\section{Funding}

This work was supported by Shanghai Health Development Planning Commission Foundation (20130156).

\section{Availability of data and materials}

The datasets used and/or analysed during the current study are available from the corresponding author on reasonable request.

\section{Ethics approval and consent to participate}

This study was approved by the Review Board of the Shanghai First People's Hospital under the supervision of the Ethics Committee, and all patients signed the informed consent forms. 


\section{Consent for publication \\ Not applicable.}

\section{Competing interests}

The authors declare that they have no competing interests.

\section{Author details}

1 Gynecology Department, Shanghai General Hospital of Nanjing Medical University, Shanghai 200080, China. ${ }^{2}$ Gynecology Department, Changning Maternity and Infant Health Hospital, Shanghai 200051, China. ${ }^{3}$ School of Basic Medical Sciences of Nanjing Medical University, Nanjing 211166, China.

Received: 9 February 2019 Accepted: 1 July 2019

Published online: 11 July 2019

\section{References}

1. Jemal A, Siegel R, Ward E, Hao Y, Xu J, Murray T, Thun MJ. Cancer statistics, 2008. CA Cancer J Clin. 2008;58(2):71-96.

2. Shintani T, Klionsky DJ. Autophagy in health and disease: a double-edged sword. Science. 2004;306(5698):990-5.

3. Paillaud JL, Harbuzaru B, Patarin J, Bats N. Extra-large-pore zeolites with two-dimensional channels formed by 14 and 12 rings. Science. 2004;304(5673):990-2.

4. Sun CY, Zhang QY, Zheng GJ, Feng B. Autophagy and its potent modulators from phytochemicals in cancer treatment. Cancer Chemother Pharmacol. 2019;83(1):17-26.

5. Feng H, Cheng X, Kuang J, Chen L, Yuen S, Shi M, Liang J, Shen B, Jin Z, Yan J, et al. Apatinib-induced protective autophagy and apoptosis through the AKT-mTOR pathway in anaplastic thyroid cancer. Cell Death Dis. 2018;9(10):1030.

6. Hu MB, Zhang JW, Gao JB, Qi YW, Gao Y, Xu L, Ma Y, Wei ZZ. Atorvastatin induces autophagy in MDA-MB-231 breast cancer cells. Ultrastruct Pathol. 2018;42(5):409-15.

7. Zhang Y, Liu S, Feng Q, Huang X, Wang X, Peng Y, Zhao Z, Liu Z. Perilaldehyde activates AMP-activated protein kinase to suppress the growth of gastric cancer via induction of autophagy. J Cell Biochem. 2018;120:1716-25.

8. Ahumada-Castro U, Silva-Pavez E, Lovy A, Pardo E, Molgomicron J, Cardenas C. MTOR-independent autophagy induced by interrupted endoplasmic reticulum-mitochondrial $\mathrm{Ca}(2+)$ communication: a dead end in cancer cells. Autophagy. 2019;15(2):358-61.

9. Song F, Li L, Liang D, Zhuo Y, Wang X, Dai H. Knockdown of long noncoding RNA urothelial carcinoma associated 1 inhibits colorectal cancer cell proliferation and promotes apoptosis via modulating autophagy. J Cell Physiol. 2019;234(5):7420-34.

10. Martin KR, Celano SL, Solitro AR, Gunaydin H, Scott M, O'Hagan RC, Shumway SD, Fuller P, MacKeigan JP. A potent and selective ULK1 inhibitor suppresses autophagy and sensitizes cancer cells to nutrient stress. iscience. 2018:8:74-84.

11. Yang F, Wang F, Liu Y, Wang S, Li X, Huang Y, Xia Y, Cao C. Sulforaphane induces autophagy by inhibition of HDAC6-mediated PTEN activation in triple negative breast cancer cells. Life Sci. 2018;213:149-57.

12. Yang K, Niu T, Luo M, Tang L, Kang L. Enhanced cytotoxicity and apoptosis through inhibiting autophagy in metastatic potential colon cancer SW620 cells treated with Chlorin e6 photodynamic therapy. Photodiagn Photodyn Ther. 2018;24:332-41.

13. Arya BD, Mittal S, Joshi P, Pandey AK, Ramirez-Vick JE, Singh SP. Graphene oxide-chloroquine nanoconjugate induce necroptotic death in A549 cancer cells through autophagy modulation. Nanomedicine. 2018;13(18):2261-82

14. Chen HE, Lin JF, Tsai TF, Lin YC, Chou KY, Hwang TI. Allyl isothiocyanate induces autophagy through the up-regulation of beclin-1 in human prostate cancer cells. Am J Chin Med. 2018;46:1-19.

15. Zhu J, Zhao B, Xiong P, Wang C, Zhang J, Tian X, Huang Y. Curcumin induces autophagy via inhibition of yes-associated protein (YAP) in human colon cancer cells. Med Sci Monitor. 2018:24:7035-42.
16. Jiao YN, Wu LN, Xue D, Liu XJ, Tian ZH, Jiang ST, Han SY, Li PP. Marsdenia tenacissima extract induces apoptosis and suppresses autophagy through ERK activation in lung cancer cells. Cancer Cell Int. 2018;18:149.

17. Batool S, Joseph TP, Hussain M, Vuai MS, Khinsar KH, Din SRU, Padhiar AA, Zhong M, Ning A, Zhang W, et al. LP1 from lentinula edodes C91-3 induces autophagy, apoptosis and reduces metastasis in human gastric cancer cell line SGC-7901. Int J Mol Sci. 2018;19(10):2986.

18. Zhao Z, Xia G, Li N, Su R, Chen X, Zhong L. Autophagy inhibition promotes bevacizumab-induced apoptosis and proliferation inhibition in colorectal cancer cells. J Cancer. 2018;9(18):3407-16.

19. Shi Y, Zhang B, Feng X, Qu F, Wang S, Wu L, Wang X, Liu Q, Wang P, Zhang K. Apoptosis and autophagy induced by DVDMs-PDT on human esophageal cancer Eca-109 cells. Photodiagn Photodyn Ther. 2018;24:198-205.

20. Wang FY, Huang KB, Feng HW, Chen ZF, Liu YN, Liang H. New Platinum(II) agent induces bimodal death of apoptosis and autophagy against A549 cancer cell. Free Radical Biol Med. 2018;129:418-29.

21. Hu YJ, Zhang JY, Luo Q, Xu JR, Yan Y, Mu LM, Bai J, Lu WL. Nanostructured dihydroartemisinin plus epirubicin liposomes enhance treatment efficacy of breast cancer by inducing autophagy and apoptosis. Nanomaterials. 2018;8(10):804.

22. Kurarmoto H, Hamano M, Imai M. HEC-1 cells. Hum Cell. 2002;15(2):81-95

23. Agarwal V, Bell GW, Bartel DP. Nam JW. eLife: Predicting effective microRNA target sites in mammalian mRNAs; 2015. p. 4.

24. Betel D, Koppal A, Agius P, Sander C, Leslie C. Comprehensive modeling of microRNA targets predicts functional non-conserved and non-canonical sites. Genome Biol. 2010;11(8):R90.

25. Khan A, Mathelier A. Intervene: a tool for intersection and visualization of multiple gene or genomic region sets. BMC Bioinform. 2017;18(1):287.

26. Zou Y, Yao S, Chen X, Liu D, Wang J, Yuan X, Rao J, Xiong H, Yu S, Yuan $X$, et al. LncRNA OIP5-AS1 regulates radioresistance by targeting DYRK1A through miR-369-3p in colorectal cancer cells. Eur J Cell Biol. 2018;97(5):369-78.

27. Li P, Dong M, Wang Z. Downregulation of TSPAN13 by miR-369-3p inhibits cell proliferation in papillary thyroid cancer (PTC). Bosn J Basic Med Sci. 2019;19(2):146-54.

28. Jo YK, Roh SA, Lee H, Park NY, Choi ES, Oh JH, Park SJ, Shin JH, Suh YA, Lee EK, et al. Polypyrimidine tract-binding protein 1-mediated down-regulation of ATG10 facilitates metastasis of colorectal cancer cells. Cancer Lett. 2017;385:21-7

29. Liu P, Wang C, Ma C, Wu Q, Zhang W, Lao G. MicroRNA-23a regulates epithelial-to-mesenchymal transition in endometrial endometrioid adenocarcinoma by targeting SMAD3. Cancer Cell Int. 2016;16(1):67.

30. Canlorbe G, Wang Z, Laas E, Bendifallah S, Castela M, Lefevre M, ChabbertBuffet N, Darai E, Aractingi S, Mehats C, et al. Identification of microRNA expression profile related to lymph node status in women with earlystage grade 1-2 endometrial cancer. Mod Pathol. 2016;29(4):391-401.

31. Lee TS, Jeon HW, Kim YB, Kim YA, Kim MA, Kang SB. Aberrant microRNA expression in endometrial carcinoma using formalin-fixed paraffinembedded (FFPE) tissues. PLoS ONE. 2013:8(12):e81421.

32. Wilczynski M, Danielska J, Domanska-Senderowska D, Dzieniecka M, Szymanska B, Malinowski A. Association of microRNA-200c expression levels with clinicopathological factors and prognosis in endometrioid endometrial cancer. Acta Obstet Gynecol Scand. 2018:97(5):560-9.

33. Hao GJ, Ding YH, Wen H, Li XF, Zhang W, Su HY, Liu DM, Xie NL. Attenuation of deregulated miR-369-3p expression sensitizes non-small cell lung cancer cells to cisplatin via modulation of the nucleotide sugar transporter SLC35F5. Biochem Biophys Res Commun. 2017;488(3):501-8.

34. Guo S, Zhang W, Wei C, Wang L, Zhu G, Shi Q, Li S, Ge R, Li K, Gao L, et al. Serum and skin levels of miR-369-3p in patients with psoriasis and their correlation with disease severity. Eur J Dermatol EJD. 2013;23(5):608-13.

35. Pan W, Yu H, Zheng B, Gao Y, Li P, Huang Q, Xie C, Ge X. Upregulation of MiR-369-3p suppresses cell migration and proliferation by targeting SOX4 in Hirschsprung's disease. J Pediatr Surg. 2017;52(8):1363-70.

\section{Publisher's Note}

Springer Nature remains neutral with regard to jurisdictional claims in published maps and institutional affiliations. 